Document downloaded from:

http://hdl.handle.net/10251/60381

This paper must be cited as:

Debón Aucejo, AM.; Martinez Ruiz, F.; Montes, F. (2010). A geostatistical approach for dynamic life tables: The effect of mortality on remaining lifetime and annuities. Insurance: Mathematics and Economics. 47(3):327-336. doi:10.1016/j.insmatheco.2010.07.007.

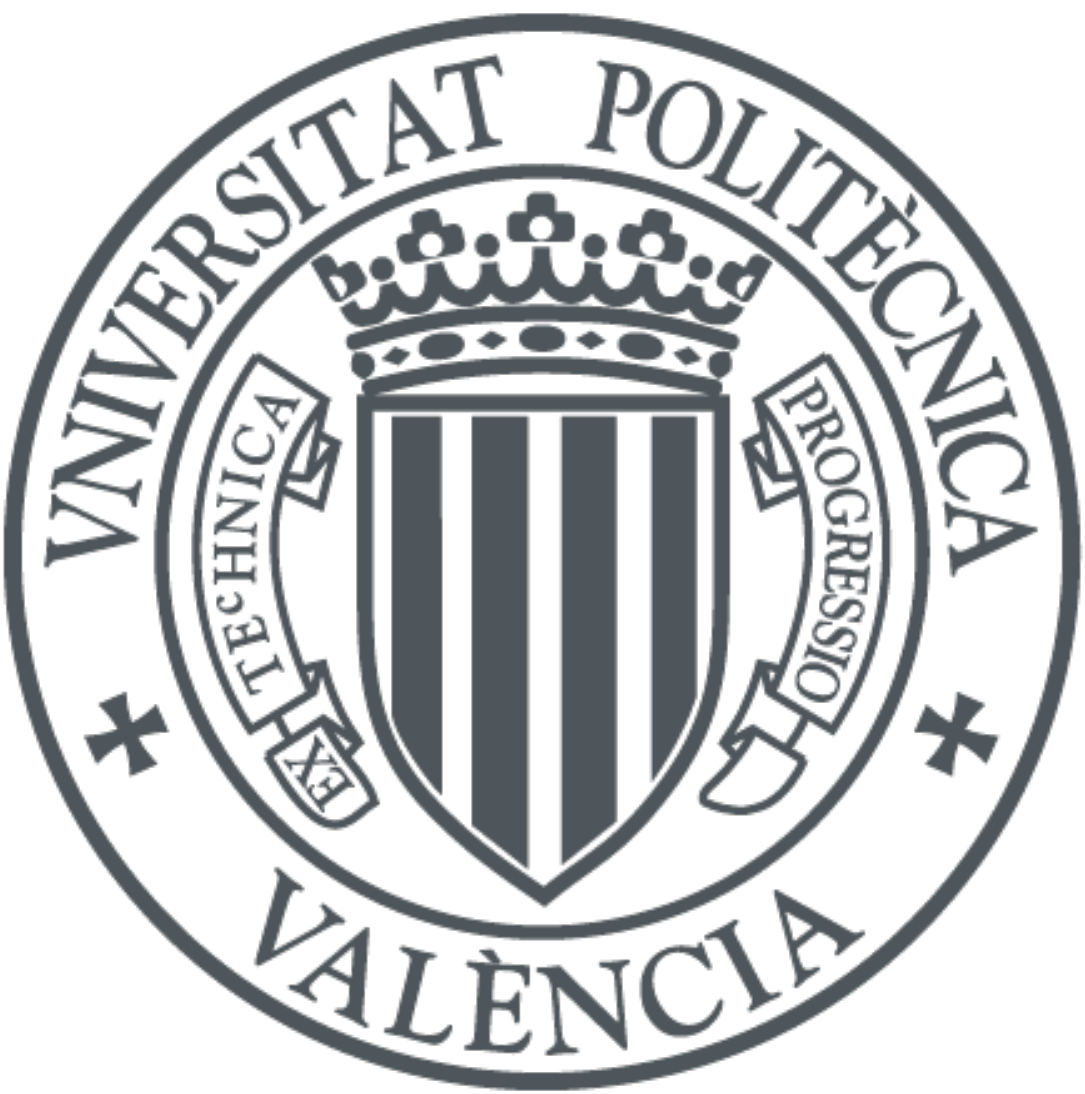

The final publication is available at

http://dx.doi.org/10.1016/j.insmatheco.2010.07.007

Copyright Elsevier

Additional Information 


\title{
A Geostatistical Approach for Dynamic Life Tables. The effect of mortality on remaining lifetime and annuities
}

\author{
A. Debón ${ }^{\mathrm{a}}$, F. Martínez-Ruiz ${ }^{\mathrm{b}}$, F. Montes ${ }^{\mathrm{b}}$ \\ ${ }^{a}$ e-mail: andeau@eio.upv.es. Centro de Gestión de la Calidad y del Cambio. Universidad \\ Politécnica de Valencia. Camino de Vera s/n. 46022 Valencia. Spain \\ ${ }^{b}$ Dpt. d'Estadística i I. O. Universitat de València. Spain
}

\begin{abstract}
Dynamic life tables arise as an alternative to the standard (static) life table with the aim of incorporating the evolution of mortality over time. The parametric model introduced by Lee and Carter in 1992 for projected mortality rates in the US is one of the most outstanding and has been largely used since then. Different versions of the model have been developed but all of them, together with other parametric models, consider the observed mortality rates as independent observations. This is a difficult hypothesis to hold when looking at the graph of the residuals obtained with any of these methods.
\end{abstract}

Methods of adjustment and prediction based on geostatistical techniques which exploit the dependence structure existing among the residuals are an alternative approach to classical methods. Dynamic life tables can be considered as a two-way table on a grid equally spaced in either the vertical (age) or horizontal (year) direction, and the data can be decomposed into a deterministic large-scale variation (trend) plus a stochastic small-scale variation (residuals). 
Our contribution consists of applying geostatistical techniques for estimating the dependence structure of the mortality data and for prediction purposes, also including the influence of the year of birth (cohort). We compare the performance of this new approach with different versions of the Lee-Carter model. Additionally, we obtain bootstrap confidence intervals for predicted $q_{x t}$ resulting from applying both methodologies, and we study their influence on the predictions of $e_{65 t}$ and $a_{65 t}$.

Key words: Life Table, Geostatistics, Bootstrap Confidence Intervals

\section{Introduction}

In static life tables the influence of age on data graduation has been widely addressed in papers such as Forfar et al. (1988), Renshaw (1991) and Debón et al. (2005), using parametric models, or in Gavin et al. (1993, 1994, 1995) and Debón et al. (2006b), who propose non-parametric models. None of these models take into account the fact that mortality progresses over the years, as they were designed to analyse data corresponding to one year in particular or, in the case of several years, to work with aggregated data.

The concept of a dynamic life table seeks to solve this problem by jointly analyzing mortality data corresponding to a series of consecutive years. This approach allows the calendar effect influence on mortality to be studied. A sample of the models developed for graduating dynamic tables can be found in Benjamin and Soliman (1993); Tabeau et al. (2001); Pitacco (2004); Wong-Fupuy and Haberman (2004); Debón et al. (2006a). Most of them adapt traditional laws to the new situation and all them share a common hypothesis: they consider the observed measures of mortality as independent 
across ages and over time. As Booth et al. (2002) point out, it is difficult to hold such a hypothesis when looking at the graph of the residuals obtained after the adjustment with any of these models.

Tools appropriate to other disciplines can be used to overcome the problem which residual dependency supposes. We have turned to Geostatistics, which provides techniques for modelling the dependency structure among a set of neighbouring observations. Covariance functions and variogram are the essential tools which, together with kriging techniques, also allow predictions to be made and associated errors to be calculated (Matheron, 1975; Journel and Huijbregts, 1978).

Geostatistical techniques were designed for the analysis of data which were very far from what a dynamic table represents. This distance is only apparent as a dynamic table can actually be considered as a set of data over a rectangular grid equally spaced both vertically, for age, and horizontally, for year. The diagonals of this grid stand for the cohort determined by the age and the year. On the other hand, the aim of Geostatistics is, as already mentioned, to model the dependence structure among neighbours, which requires defining a neighbourhood relationship as well as a distance. They are straightforward in the case of spatial data but also possible in other kind of data. The analysis of sudden infant death syndrome (SIDS) in North Carolina in Cressie (1993), as well as the analysis using spatial techniques of the 1970 US Draft Lottery (Mateu et al., 2004), support this assessment. Moreover, as in previous studies, we will show that these methods provide better solutions than the classical methods since they simultaneously take into account the effect of age and time, while the others treat both effects 
separately.

This article is structured as follows: Section 2 introduces the original Lee-Carter model with one and two time terms and the Lee-Carter ageperiod-cohort model, derived from the original one but adding a second term for collecting the influence of cohort over mortality. This section ends with an introduction to geostatistical methodology, including a brief description of the median polish algorithm that will be used for estimating the deterministic trend of the geostatistic model proposed. Section 3 briefly presents the bootstrapping techniques used for obtaining confidence intervals. Section 4 presents the results of the application of the seven models, three Lee-Carter and four median polish models, to the analysis of mortality data in Spain for the period 1980-2003. The results of the prediction of $q_{x, 2004}$ and $q_{x, 2005}$, death probability at age $x$ in year 2004 and 2005, obtained from the projections of the adjusted models, are also collected in this section. The last part of Section 4 is devoted to obtaining the confidence intervals for the prediction of residual life expectancy and the annuities, $e_{65 t}$ and $a_{65 t}$. The results which provide the distinct models for the period 2004-2023 are compared, the evolution of the annuities being of particular interest for, as far as we know, we have not found evidence of any other similar study on mortality data in Spain. Finally, Section 5 establishes the conclusions to be drawn from the results in the previous section.

\section{Adjustment and prediction of mortality rates}

We consider a set of crude mortality rates $\dot{q}_{x t}$, for age $x \in\left[x_{1}, x_{k}\right]$ and

calendar year $t \in\left[t_{1}, t_{n}\right]$, which we use to produce smoother estimates, $\hat{q}_{x t}$, of 
the true but unknown mortality probabilities $q_{x t}$. A crude rate at age $x$ and time $t$ is typically based on the corresponding number of deaths recorded, $d_{x t}$, relative to those initially exposed to risk, $E_{x t}$.

\subsection{Lee-Carter models}

The Lee-Carter Model, developed in Lee and Carter (1992), consists of adjusting the following function to the measure of mortality ,

$$
m_{x t}=\exp \left(a_{x}+b_{x} k_{t}+\epsilon_{x t}\right)
$$

or, equally, the function

$$
\ln \left(m_{x t}\right)=a_{x}+b_{x} k_{t}+\epsilon_{x t}
$$

applied to its logarithm transform. This is an age-period (AP) model as the double subscript refers to the age, $x$, and to the year or unit of time, $t$. In (1) and (2), $a_{x}$ and $b_{x}$ are age-dependent parameters and $k_{t}$ is a specific mortality index for each year or unit of time. The errors $\epsilon_{x t}$, with a zero average and variance $\sigma_{\epsilon}^{2}$, reflect the historical influences of each specific age that are not captured by the model.

Various authors have proposed modifications to the Lee-Carter model. Booth et al. (2002) and Renshaw and Haberman (2003) propose the inclusion of a new term in (2), of the form $b_{x} k_{t}$, with the objective of improving the fit and trying to eliminate the trend shown by the residuals with the original model. Renshaw and Haberman (2006) propose an age-cohort model (AC) in which the time scale $t$ of the original model is substituted by the cohort $c=t-x$. In the same article they also propose an age-period-cohort (APC) 
which adds a new term to (2) which, different from what is suggested in Booth et al. (2002), includes the influence of the cohort on mortality.

All these variations on the original Lee-Carter model can be expressed through a unified model whose expression, for the logit of the mortality ratio which we are going to work with, is

$$
\ln \left(\frac{q_{x u}}{1-q_{x u}}\right)=a_{x}+b_{x}^{1} k_{u_{1}}+b_{x}^{2} k_{u_{2}}+\epsilon,
$$

where $a_{x}, b_{x}^{1}$ and $b_{x}^{2}$ are age-dependent parameters and $k_{u_{1}}$ and $k_{u_{2}}$ are specific mortality indexes for each unit of the time scale. The errors $\epsilon$, with a zero average and variance $\sigma_{\epsilon}^{2}$, reflect the historical influences of each specific age that are not captured by the model. Table 1 shows the expressions of the distinct models and, in brackets, the abbreviated names with which they are identified later on.

\section{Model Parameters Expression}

$\begin{array}{lll}\text { A-P (LC) } & u_{1}=t, b_{x}^{2}=0 & \ln \left(\frac{q_{x t}}{1-q_{x t}}\right)=a_{x}+b_{x} k_{t}+\epsilon_{x t} \\ \text { A-P 2 (LC2) } & u_{1}=t, u_{2}=t & \ln \left(\frac{q_{x t}}{1-q_{x t}}\right)=a_{x}+b_{x}^{1} k_{t}^{1}+b_{x}^{2} k_{t}^{2}+\epsilon_{x t}, \\ \text { A-C (LCC) } & u_{1}=c, b_{x}^{2}=0 & \ln \left(\frac{q_{x t}}{1-q_{x t}}\right)=a_{x}+b_{x} k_{c}+\epsilon_{x t} \\ \text { A-P-C (LC-APC) } & u_{1}=t, u_{2}=c & \ln \left(\frac{q_{x t}}{1-q_{x t}}\right)=a_{x}+b_{x}^{1} k_{t}+b_{x}^{2} k_{c}+\epsilon_{x t} .\end{array}$

Table 1: Four versions of Lee-Carter model 
All these models, except the LCC, will be used to adjust the Spanish mortality data described later on. The adjustment is carried out through ML using the gnm library (Turner and Firth, 2006) of the R Development Core Team (2005). In the Appendix details are added regarding the necessary normalisation of parameters and, in particular, how to overcome the problem which appears in the LC-APC model due to the relationship which exits between age, period and the cohort, $c=t-x$.

\subsection{Geostatistical methods}

The mortality data we want to analyze can be considered as a set of spatio-temporal data, with age as the unidimensional spatial component and year as the temporal one.

Following Cressie and Majure (1997), we denote by $Z(x, t)$ the mortality measure at age $x$ and time $t$. We suppose that we have observed the data $\{Z(x, t), x \in D, t \in T\}$, and we wish to predict the process value $Z\left(x_{0}, t_{0}\right)$ at the age $x_{0} \in D$ for the year $t_{0}$. In order to achieve the convenient stationarity, we assume that the data can be decomposed into a deterministic large-scale variation (trend) plus a stochastic small-scale variation (error). The model can be written as,

$$
Z(x, t)=\mu(x, t)+\delta(x, t)
$$

where $E[Z(x, t)]=\mu(x, t)$ and $\delta(\cdot, \cdot)$ is a zero-mean second order stationary Gaussian process with covariance function

$$
C(h, u)=\operatorname{Cov}[Z(x+h, t+u), Z(x, t)]
$$

and variogram

$$
2 \gamma(h, u)=\operatorname{Var}[Z(x+h, t+u)-Z(x, t)]=\operatorname{Var}[\delta(x+h, t+u)-\delta(x, t)],
$$


both characterizing the spatial and temporal dependence. In fact, under the hypothesis of second order stationarity, the variogram and covariance function are related throughout

$$
2 \gamma(h, u)=2 C(0,0)-2 C(h, u)
$$

The large-scale variation $\mu(x, t)$ and the small-scale variation $\delta(\cdot, \cdot)$ are modelled respectively as deterministic and stochastic processes, but there is no way of making the decomposition identifiable.

The error process $\delta(x, t)$ can be estimated by subtracting the estimation of $\mu(x, t)$ from $(4) \hat{\mu}(x, t)$,

$$
\hat{\delta}(x, t)=Z(x, t)-\hat{\mu}(x, t)
$$

The estimation of the covariance function can be obtained using the classical estimator based on the moments method (Cressie (1993), Sec.2.4),

$$
\hat{C}(h, u)=\frac{1}{|N(h, u)|} \sum_{|N(h, u)|}\left(\hat{\delta}\left(x_{i}, t_{i}\right)-m_{\hat{\delta}}\right)\left(\hat{\delta}\left(x_{j}, t_{j}\right)-m_{\hat{\delta}}\right)
$$

where,

$$
N(h, u)=\left\{\left(\left(x_{i}, t_{i}\right),\left(x_{j}, t_{j}\right)\right), x_{i}-x_{j}=h, t_{i}-t_{j}=u\right\}
$$

$|N(h, u)|$ is the number of distinct pairs in $N(h, u)$ and $m_{\hat{\delta}}$ is the sample mean of observed residuals.

The problem with the empirical covariance function is that the conditions of validity are not satisfied (Cressie, 1993), so a valid model is fitted to the empirical one using the weighted least squares criterion. The variogram is now easily obtained from (5). 
Once this is done, we can estimate the original process value $Z\left(x_{0}, t_{0}\right)$ with the large-scale process estimated value $\hat{\mu}\left(x_{0}, t_{0}\right)$, plus the error process estimated value $\hat{\delta}\left(x_{0}, t_{0}\right)$, obtaining an original process value prediction $\hat{Z}\left(x_{0}, t_{0}\right)$ and its mean-squared prediction error.

For the prediction of $\hat{\delta}\left(x_{0}, t_{0}\right)$ from the $n$ data $\{\hat{\delta}(x, t), x \in D, t \in T\}$, we used the ordinary kriging approach. This method uses a linear predictor,

$$
\hat{p}_{\delta}\left(x_{0}, t_{0}\right)=\sum_{i=1}^{n} \lambda_{i} \hat{\delta}\left(x_{i}, t_{i}\right)=\boldsymbol{\lambda}^{\prime} \hat{\boldsymbol{\delta}}
$$

with $\boldsymbol{\lambda}^{\prime} \mathbf{1}=\sum_{i=1}^{n} \lambda_{i}=1$ to guarantee uniform unbiasedness.

The $\boldsymbol{\lambda}$ values that minimize the mean-squared prediction error subject to this constraint are,

$$
\boldsymbol{\lambda}_{o k}^{\prime}=\left(\gamma+1 \frac{1-\mathbf{1}^{\prime} \boldsymbol{\Gamma}^{-1} \gamma}{\mathbf{1}^{\prime} \boldsymbol{\Gamma}^{-1} \mathbf{1}}\right)^{\prime} \Gamma^{-1}
$$

where $\boldsymbol{\gamma}=\left(\gamma\left(\left|x_{0}-x_{1}\right|,\left|t_{0}-t_{1}\right|\right) \ldots \gamma\left(\left|x_{0}-x_{n}\right|,\left|t_{0}-t_{n}\right|\right)\right)^{\prime}$, and $\boldsymbol{\Gamma}$ is the $n \times n$ matrix whose $(i, j)$-th element is $\gamma\left(\left|x_{i}-x_{j}\right|,\left|t_{i}-t_{j}\right|\right)$.

The minimized mean square prediction error (or kriging variance) is given by,

$$
\sigma_{o k}^{2}\left(x_{0}, t_{0}\right)=\gamma^{\prime} \boldsymbol{\Gamma}^{-1} \gamma-\frac{\left(\mathbf{1}^{\prime} \boldsymbol{\Gamma}^{-1} \boldsymbol{\gamma}-1\right)^{2}}{\mathbf{1}^{\prime} \boldsymbol{\Gamma}^{-1} \mathbf{1}}
$$

Finally, the optimal prediction for the original process value is,

$$
\hat{p}_{z}\left(x_{0}, t_{0}\right)=\hat{Z}\left(x_{0}, t_{0}\right)=\hat{\mu}\left(x_{0}, t_{0}\right)+\hat{p}_{\delta}\left(x_{0}, t_{0}\right)
$$

and its standard error,

$$
\sigma_{z}\left(x_{0}, t_{0}\right)=\sigma_{o k}\left(x_{0}, t_{0}\right)
$$




\subsubsection{Data transformation}

The mortality data, proportions or probabilities, do not satisfy the normality and stationarity conditions and a transformation of the original process is needed to achieve them (Cressie, 1993). When this occurs, and the transformation is

$$
Y(x, t)=\phi(Z(x, t))
$$

with $\phi^{-1}$ twice-differentiable, a bias corrected optimal prediction $\hat{Z}\left(x_{0}, t_{0}\right)$ is given by

$$
\check{p}_{z}\left(x_{0}, t_{0}\right)=\phi^{-1}\left(\hat{p}_{y}\left(x_{0}, t_{0}\right)\right)+\left(\phi^{-1}\right)^{\prime \prime}\left(\tilde{\mu}_{y}\left(x_{0}, t_{0}\right)\right)\left\{\sigma_{y}^{2}\left(x_{0}, t_{0}\right) / 2-m_{y}\right\}
$$

where $m_{y}$ are the Lagrange multiplier of the ordinary kriging equation,

$$
m_{y}=-\frac{1-\mathbf{1}^{\prime} \boldsymbol{\Gamma}^{-1} \boldsymbol{\gamma}}{\mathbf{1}^{\prime} \boldsymbol{\Gamma}^{-1} \mathbf{1}}
$$

The mean square prediction error is then approximated by

$$
\sigma_{z}^{2}\left(x_{0}, t_{0}\right)=\left\{\left(\phi^{-1}\right)^{\prime}\left(\tilde{\mu}_{y}\left(x_{0}, t_{0}\right)\right)\right\}^{2} \sigma_{y}^{2}\left(x_{0}, t_{0}\right) .
$$

\subsubsection{Modelling trend}

Lee-Carter models.- The modelling of the deterministic trend in (4), $\mu(x, t)$, can be carried out through any of the Lee-Carter models described in Section 2.1, subsequently adjusting a covariance function to the residue resulting in (6). Specifically, as was pointed out at the end of Section 2.1, only the models denominated LC, LC2 and LC-APC will be used to adjust the trend, giving rise to three new models which we will denominate in the same way as the originals, but adding the suffix res. 
Median Polish.- Another option for modelling trend is to consider the mortality data in a dynamic life table as a two-way table. In fact, if the observations were independent, a two factor age and year ANOVA could be applied in order to analyze them. An alternative is to consider this two-way table as a rectangular grid equally spaced in either the vertical (age) or horizontal (year) direction. In this context, the deterministic component in $(4), \mu(x, t)$, can be expressed as the sum

$$
\mu(x, t)=\mu+r_{x}+c_{t},
$$

where $\mu$ is an overall effect, $r_{x}$ is a row effect due to age and $c_{t}$ is a column effect due to year. Their ordinary-least-square estimators are the corresponding sample means, but some problems of bias can arise when estimating the covariance function or the variogram. These problems can be overcome using sample medians. A median polish algorithm producing overall effect, $\hat{\mu}$, row effects, $\hat{r}_{x}, x \in D$, and column effects, $\hat{c}_{t}, t \in T$, can be found in Cressie (1993).

The deterministic trend (14) is an age-period one. As in the Lee-Carter model, we can enlarge the trend introducing a cohort effect,

$$
\mu(x, t)=\mu+r_{x}+c_{t}+d_{c}
$$

for whose estimation we have developed a new version of the original median-polish algorithm.

Four new models for mortality adjustment are derived from here on. Two of them will consist of only adjusting the trend of the data, being those corresponding to the expressions (14) and (15) and which we will 
denote later on as MP and MP-APC. The other two are those which are derived from these by modelling their corresponding residuals through a valid covariance function. We will denote them as MP-res and MPAPC-res, respectively.

\subsection{Prediction for future years}

For all the models described in the previous sections, the prediction beyond the period under observation, for example for the year $t_{n}+s$, has been carried out adjusting an ARIMA model to the series of time parameters, whether they be periods or cohorts. With the time series adjusted, the parameters are projected, which once substituted in the models provide the prediction of the probabilities of death. Throughout this process the parameters dependent on age stay fixed. Prediction expressions for the distinct models are shown in Table 2 where $c^{*}=t_{n}+s-x$. This procedure supposes, in the specific case of the MP model, a difference with respect to the original treatment proposed by Cressie (1993), who predicts using only the values of the parameters of the last two periods.

Notice that models based on geostatistical techniques have a second prediction term corresponding to residuals, namely $\hat{p}_{\delta}\left(x, t_{n}+s\right)$, obtained according to $(9)$.

\section{Bootstrap confidence intervals}

Mortality predictions are not normally accompanied by measures of sensitivity and uncertainty. Some authors, Pedroza (2006) among others, argue that such measures are necessary and suggest the construction of confidence intervals for the estimations obtained. It should be recalled that Lee and 


\begin{tabular}{ll} 
Model & Prediction \\
\hline LC & $\operatorname{logit}\left(\hat{q}_{x, t_{n}+s}\right)=\hat{a}_{x}+\hat{b}_{x} \hat{k}_{t_{n}+s}$ \\
LC-res & $\operatorname{logit}\left(\hat{q}_{x, t_{n}+s}\right)=\hat{a}_{x}+\hat{b}_{x} \hat{k}_{t_{n}+s}+\hat{p}_{\delta}\left(x, t_{n}+s\right)$ \\
LC2 & $\operatorname{logit}\left(\hat{q}_{x, t_{n}+s}\right)=\hat{a}_{x}+\hat{b}_{x}^{1} \hat{k}_{t_{n}+s}^{1}+\hat{b}_{x}^{2} \hat{k}_{t_{n}+s}^{2}$, \\
LC2-res & $\operatorname{logit}\left(\hat{q}_{x, t_{n}+s}\right)=\hat{a}_{x}+\hat{b}_{x}^{1} \hat{k}_{t_{n}+s}^{1}+\hat{b}_{x}^{2} \hat{k}_{t_{n}+s}^{2}+\hat{p}_{\delta}\left(x, t_{n}+s\right)$, \\
LC-APC & $\operatorname{logit}\left(\hat{q}_{x, t_{n}+s}\right)=\hat{a}_{x}+\hat{b}_{x}^{1} \hat{k}_{t_{n}+s}+\hat{b}_{x}^{2} \hat{k}_{c^{*}}$, \\
LC-APC-res & $\operatorname{logit}\left(\hat{q}_{x, t_{n}+s}\right)=\hat{a}_{x}+\hat{b}_{x}^{1} \hat{k}_{t_{n}+s}+\hat{b}_{x}^{2} \hat{k}_{c^{*}}+\hat{p}_{\delta}\left(x, t_{n}+s\right)$, \\
MP & $\operatorname{logit}\left(\hat{q}_{x, t_{n}+s}\right)=\hat{\mu}+\hat{r}_{x}+\hat{c}_{t_{n}+s}$, \\
MP-res & $\operatorname{logit}\left(\hat{q}_{x, t_{n}+s}\right)=\hat{\mu}+\hat{r}_{x}+\hat{c}_{t_{n}+s}+\hat{p}_{\delta}\left(x, t_{n}+s\right)$, \\
MP-APC & $\operatorname{logit}\left(\hat{q}_{x, t_{n}+s}\right)=\hat{\mu}+\hat{r}_{x}+\hat{c}_{t_{n}+s}+\hat{d}_{c^{*}}$, \\
MP-APC-res & $\operatorname{logit}\left(\hat{q}_{x, t_{n}+s}\right)=\hat{\mu}+\hat{r}_{x}+\hat{c}_{t_{n}+s}+\hat{d}_{c^{*}}+\hat{p}_{\delta}\left(x, t_{n}+s\right)$. \\
\hline
\end{tabular}

Table 2: Prediction models

Carter, conscious of this necessity, in their original article construct confidence intervals for the expected remaining life time, $e_{x t}$, taking into account only forecast errors in the projected ARIMA $k_{t}$ parameters. Nevertheless, a criticism can be made of this approach as another source of error is due to sampling errors in the parameters of the Binomial model. A way to combine these two sources of uncertainty is to use bootstrapping procedures as Brouhns et al. (2005) and Koissi et al. (2006) do. 
In the case of Spain this methodology has been used by Debón et al. (2008), who obtain confidence intervals for the predictions provided by the Lee-Carter model of one or two terms. Parametric and non-parametric bootstrap techniques are used, in both cases turning to the binomial distribution, as distinct from the work by Brouhns et al. (2005) and Koissi et al. (2006) who employ the Poisson distribution. Another difference to point out are the residuals sampled in the non-parametric case, while Debón et al. (2008), sample over the residuals given by expression (18), Koissi et al. (2006) do so over the deviance .

The obtaining of the non-parametric bootstrap confidence intervals proposed in this paper supposes a change with respect to those made by Debón et al. (2008). Deviance residuals are now used, following the commentary in Renshaw y Haberman (2008), where it is affirmed that these residuals allow the maintenance of the hypothesis of the initial distribution of mortality measurement and provide more symmetrical intervals. Another difference to highlight with respect to the work by Koissi et al. (2006) is that there the observed deaths are set, whereas now the estimated deaths $\hat{d}_{x t}$ have been set, and the observed deaths, $d_{x t}$, are obtained by sampling the residual deviance. This difference is justified because, according to Renshaw y Haberman (2008), this procedure is more in line with the spirit in Efron and Tibshirani (1993) (Section 9.4).

The procedure used is the following: starting from the deviance residuals obtained by the original data, a bootstrap sample is drawn, estimated deaths are set, $\hat{d}_{x t}$, and the observed deaths are obtained, $d_{x t}$, from the expression of those residuals for a Binomial distribution which is the one we assumed 
for the deaths, $D_{x t} \sim B\left(E_{x t}, q_{x t}\right)$. We will have

$$
r_{d e v_{x t}}=\operatorname{sign}\left(d_{x t}-\hat{d}_{x t}\right) \sqrt{2\left[d_{x t} \log \left(\frac{d_{x t}}{\hat{d_{x t}}}\right)+\left(E_{x t}-d_{x t}\right) \log \left(\frac{E_{x t}-d_{x t}}{E_{x t}-\hat{d}_{x t}}\right)\right]}
$$

or its equivalent,

$$
r_{d e v_{x t}}^{2}=2\left[d_{x t} \log \left(\frac{d_{x t}}{\hat{d}_{x t}}\right)+\left(E_{x t}-d_{x t}\right) \log \left(\frac{E_{x t}-d_{x t}}{E_{x t}-\hat{d}_{x t}}\right)\right],
$$

reaching the solution through numerical methods, for which we turn to the uniroot function from the library stats of $\mathrm{R}$.

With the new deaths observed, the new crude mortality ratios are obtained, and thereafter a new adjustment of the model which provides new estimations of the parameters. The process is repeated for the $N$ bootstrap samples, which in turn provides a sample of size $N$ for the set of model parameters, and the $k_{t}$ 's are then projected on the basis of an ARIMA model, allowing so the obtention of prediction for mortality ratio and the corresponding life expectancy and annuities for the desired future years. The confidence intervals are obtained from the percentiles, $I C_{95}=\left[p_{0.025}, p_{0.975}\right]$.

Another change with respect to Debón et al. (2008) is that now we have also obtained bootstrap confidence intervals using geostatistical models. For that the bootstrap sample is chosen from between the logit residuals

$$
\hat{\epsilon}_{x t}^{n}=\operatorname{logit}\left(\dot{q}_{x t}\right)-\widehat{\operatorname{logit}\left(q_{x t}\right)},
$$

modelling those new errors through the appropriate covariance function. The complete model, trend plus error, allows us to predict the mortality ratio for the following year, $t_{n}+1$. The procedure is repeated for the $N$ bootstrap 
samples and the averages of the $q_{x, t_{n}+1}$ are taken as values of observed mortality corresponding to the year in question. The trend is adjusted to the widened set of observations with the data from this new year and new residual logits are obtained, the process starting over again and stopping in the last year to be predicted. The confidence intervals for the mortality averages are obtained as before, from the percentiles.

\section{Analysis of mortality data from Spain}

\subsection{Description of the data.}

The crude estimates of $q_{x t}$, necessary for the models under study, have been obtained with the process used by the Instituto Nacional de Estadística (INE, Spanish National Institute of Statistics),

$$
\dot{q}_{x, t}=\frac{1 / 2\left(d_{x, t}+d_{x, t+1}\right)}{P_{x, t}+1 / 2 d_{x, t}},
$$

where $d_{x, t}$ are deaths in the year $t$ at age $x, d_{x, t+1}$ are deaths in the year $t+1$ at age $x, \mathrm{y} P_{x, t}$ population that on December 31 of year $t$ are $x$ years old. The formula can be applied to all ages, except for zero, due to the concentration of deaths in the first few months of life. This expression has been used for zero age,

$$
\dot{q}_{0 t}=\frac{0.85 d_{0 t}+0.15 d_{0(t+1)}}{P_{0 t}+0.85 d_{0 t}} .
$$

The denominator in both expressions is an estimation of $E_{x t}$.

\subsection{Model adjustment}

The models described in Section 2 have been used to adjust mortality data in Spain for the period 1980-2003 and a range of ages from 0 to 99. The 
adjustments have been made separately for women and men.

The model performance is evaluated with three measures, the Deviance, the Mean Absolute Percentage Error $(M A P E)$ and Mean Square Error $(M S E)$. The first is a measure of the distance between observed $\dot{q}_{x t}$ and adjusted values $\hat{q}_{x t}$, whose expression is

$$
D\left(\hat{q}_{x t}\right)=2 \log L\left(\dot{q}_{x t}\right)-2 \log L\left(\hat{q}_{x t}\right)
$$

where $\log L()$ is the Binomial loglikelihood function, because we have assumed that the number of deaths is distributed as a Binomial.

The second, used by Felipe et al. (2002), is defined by,

$$
\operatorname{MAPE}\left(\hat{q}_{x t}\right)=\frac{\sum_{x} \frac{\left|\dot{q}_{x t}-\hat{q}_{x t}\right|}{\dot{q}_{x t}}}{n}, t=1, \ldots, T
$$

and it measures the mean absolute error weighted with the inverse of the crude estimates $\dot{q}_{x t}$. These weights allow us to reduce the effect of the errors associated with high values of $\dot{q}_{x t}$, usually associated with intermediate and advanced age-groups. The third, defined by,

$$
\operatorname{MSE}\left(\hat{q}_{x t}\right)=\sqrt{\sum_{x} \frac{\left(\dot{q}_{x t}-\hat{q}_{x t}\right)^{2}}{n}}, t=1, \ldots, T
$$

measures the error of estimations without any correction. The values of these three goodness of fit measures are shown in Table 3. For the parametric models, those of Lee-Carter, the respective degrees of freedom are 2178, 2079 and 2056.

Renshaw and Haberman (2006) suggest carrying out diagnostic checks on the fitted model by plotting residuals. These have been made in Figure 1 with the logit residuals (18) for some of the models. 


\begin{tabular}{l|rr|rr|rr} 
& \multicolumn{2}{|c|}{ Deviance } & \multicolumn{2}{|c|}{ MSE } & \multicolumn{2}{c}{ MAPE } \\
Model & Women & Men & Women & Men & Women & Men \\
\hline LC & 6051.85 & 14255.57 & 0.007857 & 0.009588 & 6.00 & 6.98 \\
LC2 & 3533.13 & 4598.81 & 0.004805 & 0.008588 & 4.75 & 4.19 \\
LC-APC & 2952.95 & 4272.75 & 0.005408 & 0.007360 & 4.31 & 4.12 \\
MP & 18153.05 & 24885.66 & 0.014191 & 0.013476 & 7.81 & 8.87 \\
MP-APC & 5831.11 & 13317.25 & 0.005874 & 0.008418 & 4.97 & 5.60 \\
\hline
\end{tabular}

Table 3: Goodness of fit for the different models

The predictions of mortality indexes, $\hat{k}_{t_{n}+s}, s>0$, and $\hat{k}_{c^{*}}$, have been carried out using the functions auto.arima and forecast from the $\mathrm{R}$ library forecast (Hyndman, 2008), which performs prediction automatically. These functions suppose an advantage over the previously described bootstrap procedure inasmuch as they do not require the systematic use of the ARIMA model adjusted with the original data, given that in each iteration the most adequate model is adjusted, distinct from that done by Brouhns et al. (2005); Koissi et al. (2006).

The valid covariance function adjusted to the empirical covariance function obtained from the residuals (6) is a Gneiting model, whose expression and properties are summarized in the Appendix. Figure 2 shows the empirical (left) and adjusted (right) covariance functions for the MP-res when applied to men. Observing the empirical covariance function for the LC2 and LC-APC models for women, no spatio-temporal dependency can be seen in the residuals, which implies that geostatistical modelling can not be applied, and for that reason the corresponding geostatistical models have not been 
LC
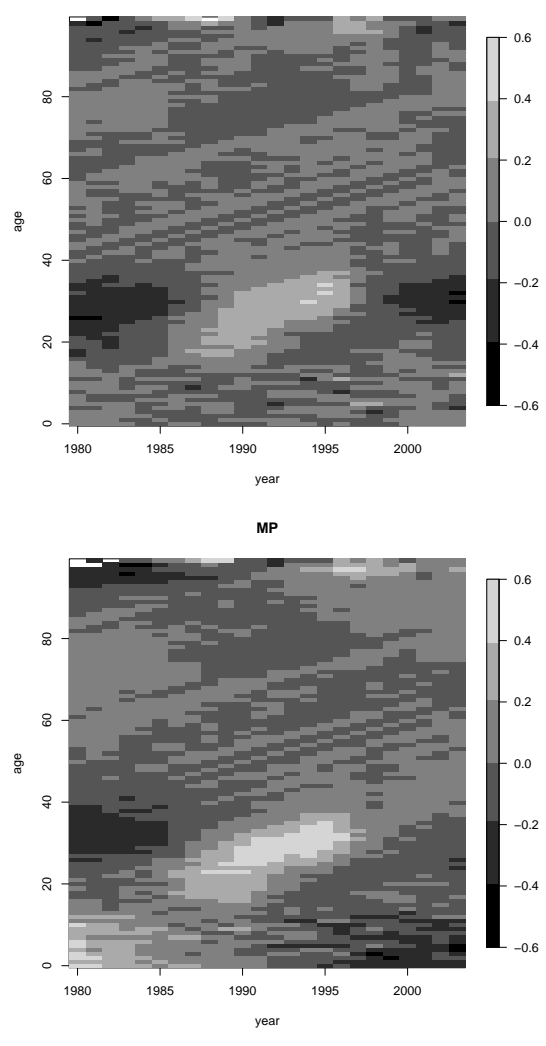

MP-APC

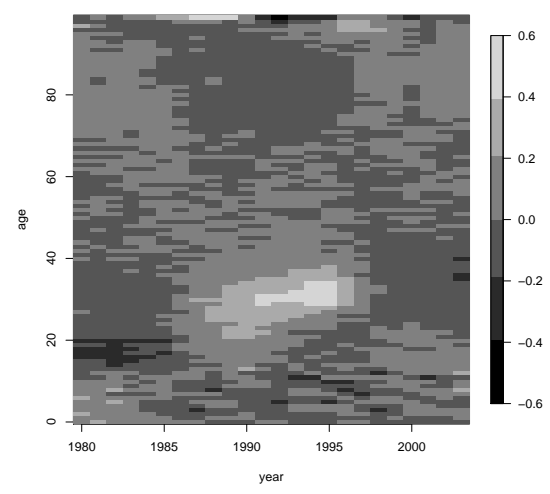

LC

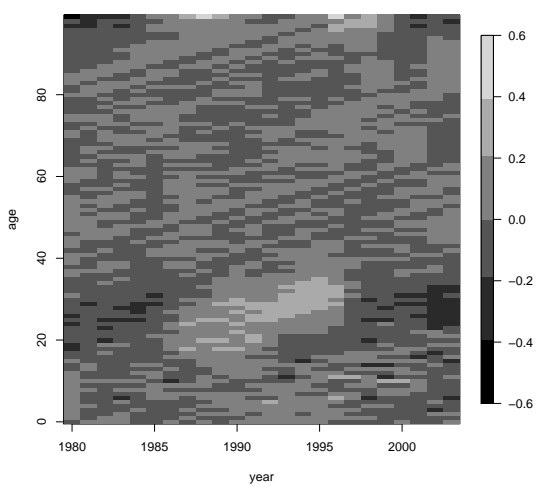

MP

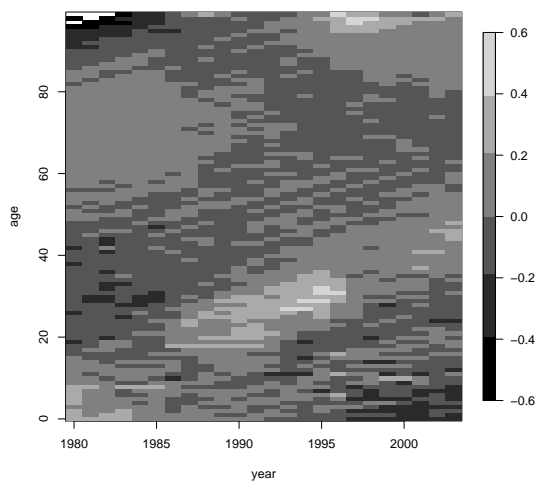

MP-APC

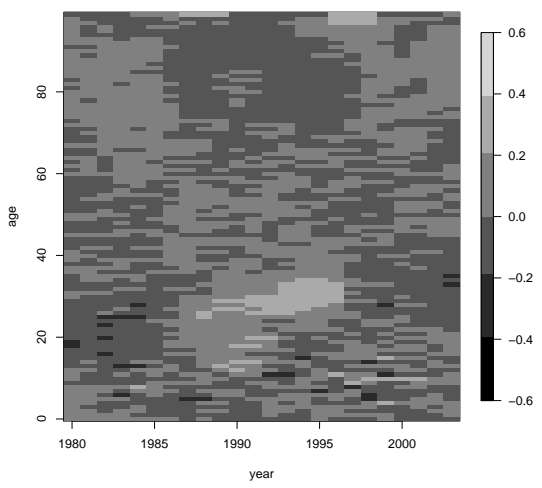

Figure 1: Residuals for age-period models for men (left) and women (right). 
adjusted.
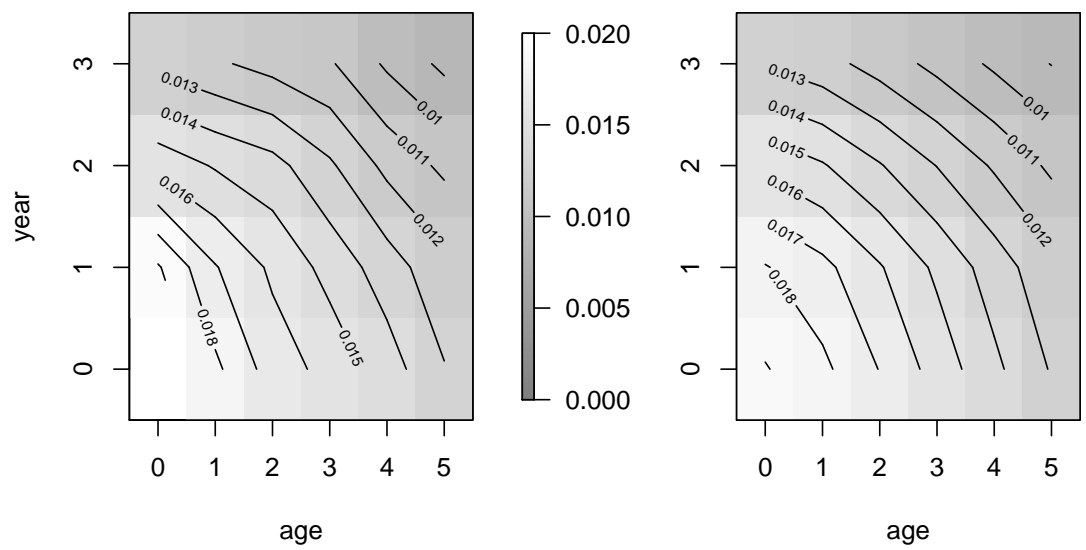

Figure 2: Empirical (left) and theoretical (right) covariance for the residuals of MP model for men.

With respect to the probabilities of death for the advanced age-group, various authors as Wong-Fupuy and Haberman (2004), warn of imprecision in the collection of data from this group, the reason why they recommend some sort of prior smoothing. We have not employed any smoothing technique in order to avoid a possible distortion in the estimation of the cohort effect.

One final comment on model adjustment. Notice that there is no information about the five Model-res either in Table 3 or in Figure 1. The reason for that is because kriging is an exact interpolator method in the absence of an error measure. Sometimes a cross-validation method can be used for estimating the goodness of fit. 


\subsection{Predictions for years 2004 and 2005}

In order to measure the goodness of prediction for each model, the predictions for $\hat{q}_{x, 2004}$ and $\hat{q}_{x, 2005}$ have been compared with the corresponding crude estimates, $\dot{q}_{x, 2004}$ and $\dot{q}_{x, 2005}$. A summary of these predictions via their residuals is presented in graph form in Figure 5, allowing a comparison of the accuracy of each method's predictions. Tables 4 and 5 show Deviance, MAPE and MSE for all the models.

\begin{tabular}{l|rr|rr|rr} 
& \multicolumn{2}{|c|}{ Deviance } & \multicolumn{2}{c|}{ MSE } & \multicolumn{2}{c}{ MAPE } \\
Model & Women & Men & Women & Men & Women & Men \\
\hline LC & 527.65 & 1403.91 & 0.0072 & 0.0086 & 11.51 & 11.94 \\
LC-res & 129.23 & 309,02 & 0.0036 & 0.0063 & 5.55 & 5.25 \\
LC2 & 157.29 & 305.06 & 0.0018 & 0.0040 & 5.77 & 4.99 \\
LC2-res & - & 182.55 & - & 0.0048 & - & 4.03 \\
LC-APC & 173.52 & 365.33 & 0.0015 & 0.0036 & 7.26 & 5.66 \\
LC-APC-res & - & 187.78 & - & 0.0035 & - & 4.31 \\
MP & 1284.56 & 1359.49 & 0.0087 & 0.0077 & 12.39 & 13.35 \\
MP-res & 154.13 & 203.84 & 0.0046 & 0.0045 & 5.66 & 4.37 \\
MP-APC & 560.30 & 1073.27 & 0.0031 & 0.0065 & 6.99 & 8.44 \\
MP-APC-res & 127.75 & 534.82 & 0.0025 & 0.0072 & 4.93 & 6.27 \\
\hline
\end{tabular}

Table 4: Goodness of fit for predictions for year 2004

\subsection{Bootstrap confidence intervals for $\boldsymbol{e}_{65 t}$ and $\boldsymbol{a}_{65 t}$}

Figures 3 and 4 shows the confidence intervals for the prediction of expected remaining lifetimes and annuities at the age of $65, e_{65 t}$ and $a_{65 t}$ for the 


\begin{tabular}{l|rr|rr|rr} 
& \multicolumn{2}{|c|}{ Deviance } & \multicolumn{2}{c|}{ MSE } & \multicolumn{2}{c}{ MAPE } \\
Model & Women & Men & Women & Men & Women & Men \\
\hline LC & 883.46 & 2091.91 & 0.0096 & 0.0110 & 14.30 & 15.53 \\
LC-res & 383.55 & 804.47 & 0.0053 & 0.0050 & 8.92 & 9.39 \\
LC2 & 324.48 & 545.71 & 0.0035 & 0.0054 & 7.52 & 6.46 \\
LC2-res & - & 512.03 & - & 0.0050 & - & 6.11 \\
LC-APC & 330.00 & 725.91 & 0.0029 & 0.0054 & 8.81 & 7.95 \\
LC-APC-res & - & 675.67 & - & 0.0065 & - & 7.08 \\
MP & 1210.48 & 1472.71 & 0.0075 & 0.0066 & 14.61 & 15.37 \\
MP-res & 386.56 & 697.87 & 0.0049 & 0.0062 & 8.71 & 8.51 \\
MP-APC & 457.55 & 1275.27 & 0.0024 & 0.0069 & 7.66 & 10.58 \\
MP-APC-res & 205.96 & 644.92 & 0.0022 & 0.0074 & 6.15 & 7.85 \\
\hline
\end{tabular}

Table 5: Goodness of fit for predictions for year 2005

period 2004-2023 obtained with different models using bootstrap techniques described in Section 3. For the left-hand graphs only the model trends have been used (only trend), and in the right-hand ones the model includes the adjustment of residuals through geostatistical methods $($ trend + res $)$.

We can see that, in general, the expected remaining lifetime is higher for women than for men and there is a clear increasing in $e_{65 t}$ over the time. This fact is true for both trend and geostatistics intervals, which show a very similar rank values for the predicted period.

Moreover, it can be seen that the MP model provides higher values for expectancy, which is due to the prediction of a reduction in the $q_{x t}$ for all ages while the other models predict increases for the advanced and interme- 
diate age groups, although these latter do not figure in the calculation of $e_{65 t}$. We should also point out the lower value of the predictions of $e_{65 t}$ obtained with the LC-APC model compared with those provided by the classic LeeCarter, LC, due to the fact that the cohorts figuring in the obtaining of the predictions increase the values of the mortality ratios. Renshaw and Haberman (2006) observe just the opposite for the mortality data for England and Wales, and we believe that the explanation for this apparent contradiction may be the distinct behaviour of Spanish mortality.

With regard to the width of the intervals, as a general observation what stands out is the narrowness of trend intervals, a feature in common with other published studies (Lee and Carter, 1992; Lee, 2000; Booth et al., 2002; Koissi et al., 2006) whose authors offer different explanations for it. In the paper by Li, Hardy y Tan (2006) the phenomenon is attributed to the rigidity of the Lee-Carter model structure and to avoid it they relax that structure by incorporating the heterogeneity from each age-period cell. As far as the influence of gender and of the model over it, there does not seem to be a clear effect of either factor when they are considered separately. We could speak, nonetheless, of the existence of an interaction between them both. For example, the greatest amplitude of intervals for the MP model for men moves to the LC-APC model for women. This comment is as valid for expected remaining lifetime as it is for the annuities. The intervals obtained with trend + res models show wider and more irregular intervals.

If we compare with similar studies carried out using Spanish mortality data, our results are slightly higher than those obtained by Guillen and Vidiella-i-Anguera (2005), though only in the case of expected remaining 
lifetime because, as we point out in the Introduction, we have not found precedents for a similar study for annuities. From these we can say that, in general, they are also higher for women than for men, in concordance with the fact that those have a higher expected remaining lifetime.
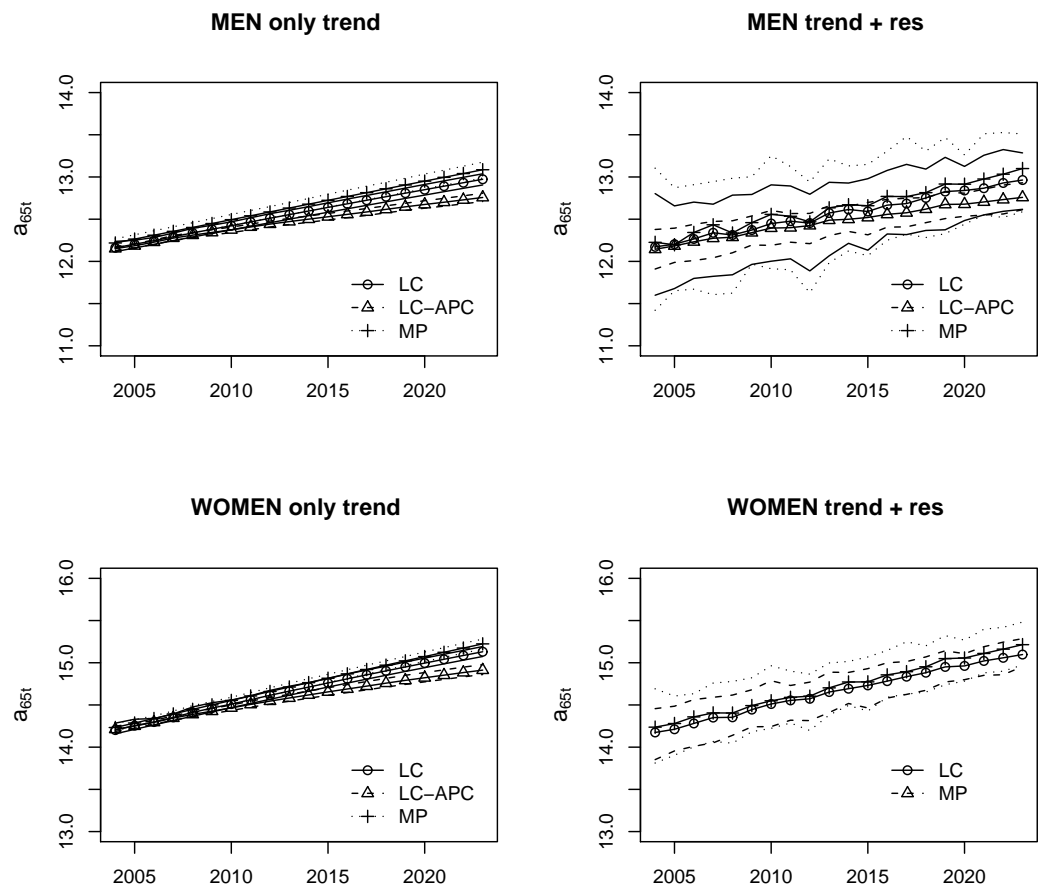

Figure 3: Bootstrap interval for $e_{65 t}$ for men and women.

\section{Conclusions}

\subsection{Model fitting}

Table 3 shows the goodness of fit values for different adjustments. A first conclusion, common to all models, is that adjustments perform better 

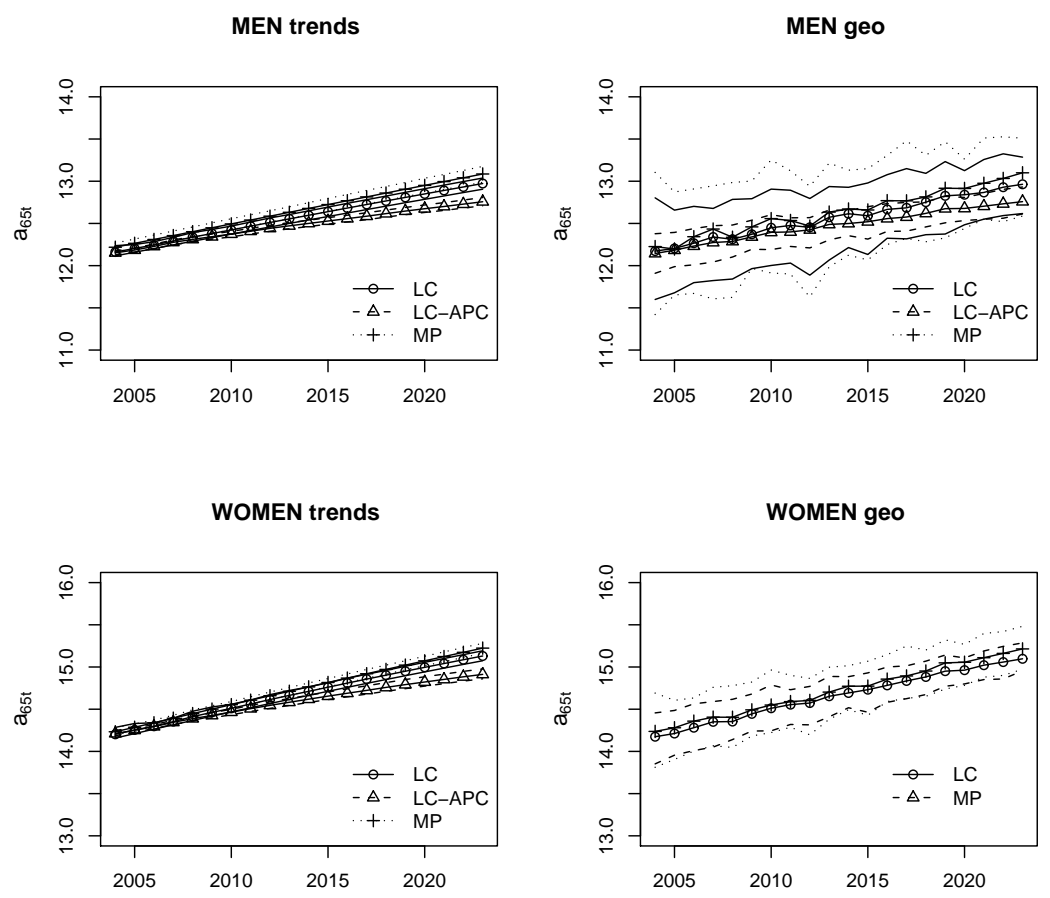

Figure 4: Bootstrap interval for $a_{65 t}$ for men and women.

for women than for men. This may be due to the fact that male mortality fluctuations for the ages in the accident hump are difficult to capture over the period of time under consideration.

The LC-APC model shows the best global result for both sexes and for the goodness-of-fit measures. The explanation of this can be found in the introduction of the cohort effect. In general, the inclusion of the cohort effect improves the models. It is worth highlighting two points: the practical disappearance in the second of the diagonal pattern which can be seen in the first, attributable to a cohort effect, and the reduction in error for intermediate 
and advanced age-groups.

\subsection{Future prediction}

Tables 4 and 5 show the results for all prediction models and also now, the first and immediate conclusion is that mortality is predicted better for women than for men.

The Geostatistical models, when applied, improve the results for both years. This improvement is pronounced for simpler models, the Lee-Carter with one term (LC) and the Media-Polish without cohort effect (MP). The LC2 and LC-APC models for women show a good performance. The explanation of this can be found in the introduction of the second term which adapts the model better for the ages involved in the accident hump (Debón et al., 2008) and for advanced and intermediate ages. In particular, the inclusion of a cohort effect improves the prediction. This better behaviour makes unnecessary to resort to geostatistics in order to model the corresponding residuals.

The greatest differences between the crude and predicted values are observed in the intermediate ages. This fact is clearly shown in Figure 5, which specifies the magnitude of residuals for all ages and models. This figure also confirms the point in the above paragraph.

A comment must be made with regard to the MP-APC model. Although it is not the model which produces the best global results, it has in its favour that its parameters are easily interpretable in as much as they describe the evolution of mortality over age, period and cohort, its computational cost is very low and it is a robust model up against the outliers. For these reasons, we think that this type of model must be borne in mind for future development. 

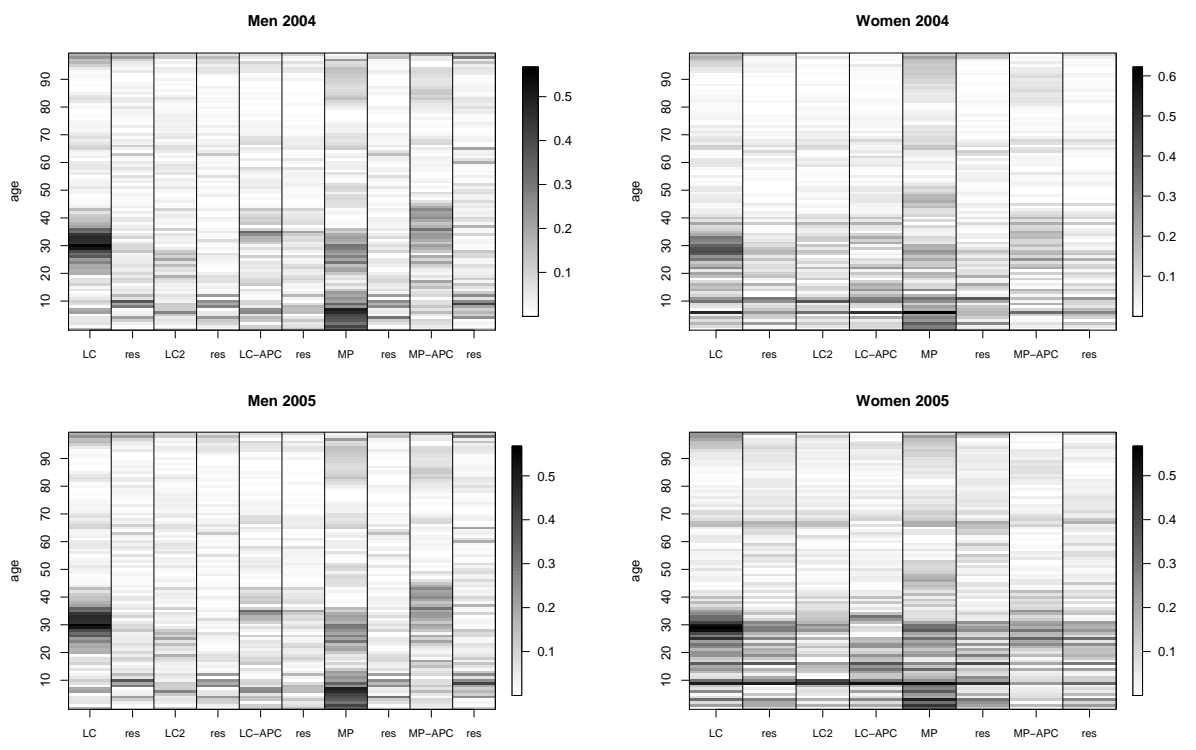

Figure 5: Absolute values of prediction residuals for each age for different models.

\subsection{A final comment}

From the preceding conclusions it can be deduced that the model with the best fit is not always the one that predicts best as, for example, the LC2 model, which generally predicts better than the LC-APC model. As Tabeau et al. (2001) affirm, good modelling is necessary for getting predictions but it does not always guarantee that these are good. On the other hand, it should be noted that in spite of a higher computational cost, LC-APC and the Geostatistical models have still a favourable cost/benefit ratio.

We must emphasise again the narrowness of the bootstrap confidence intervals obtained for expected remaining lifetime and for the annuities. Apart from the comments that this attracts from some authors and which we have referred to in Section 4.4, it can be used to deduce that the possible fluctu- 
ations in mortality ratios are not reflected in those two measures.

\section{Appendix A. Lee-Carter model fitting}

First of all, it should be pointed out that the models cannot be adjusted through normal regression methods because the values of $k_{u_{1}}$ and $k_{u_{2}}$ cannot be observed. The gnm library of the R Development Core Team (2005), developed by Turner and Firth (2006), allows adjustment. We have adapted the code written by the authors to adjust the force of mortality, $\mu_{x t}$, under a Poisson distribution and $\log$ link, to the adjustment of the probability of death, $q_{x t}$, under the Binomial distribution and logit link. In particular, its gnm function allows us to obtain generalised linear models with multilinear terms through the Mult function.

The Lee-Carter models present problems of identifiability, given a solution of $(2),\left(a_{x}, b_{x}, k_{t}\right)$, any transformation of the type $\left(a_{x}, b_{x} / c, c k_{t}\right)$ or $\left(a_{x}+\right.$ $\left.c b_{x}, b_{x}, k_{t}-c\right), \forall c$, is also a solution. In order to avoid this difficulty and to get a single solution, some constraints must be imposed to the parameters. Lee and Carter (1992) propose the normalization $\sum_{x} b_{x}=1$ and $\sum_{t} k_{t}=0$.

The Lee-Carter model with two time terms interacting with age, model LC2 in Table 1, can be adjusted with the gnm library using the instances function. The problem of identifiability in the model can be resolved with the restrictions $\sum_{x} b_{x}^{1}=1$ and $\sum_{t} k_{t}^{1}=0, \sum_{x} b_{x}^{2}=1, \sum_{t} k_{t}^{2}=0$.

The Lee-Carter model with age-period-cohort effect, model LC-APC in Table 1, presents adjustment problems due to the relationship between two factors, cohort $=$ year - age. Renshaw and Haberman (2006) propose carrying the estimation out in two stages. First, $a_{x}$ is adjusted in accordance 
with the original version by Lee-Carter,

$$
\hat{a}_{x}=\frac{\sum_{t} \ln \left(\frac{q_{x t}}{1-q_{x t}}\right)}{T} .
$$

Thereafter, the remaining parameters can be adjusted with the values of $a_{x}$ fixed through the offset term. To resolve the problem of identifiability, the authors consider the following restrictions $\sum_{x} b_{x}^{1}=1, \sum_{x} b_{x}^{2}=1$ and $k_{t_{1}}=$ 0 or $k_{c_{1}}=0$, with $t_{1}$ and $c_{1}$ being the first period and and first cohort, respectively. In line with our experience, the choice of period or origin cohort influences the convergence of the algorithm. It is worth choosing $t_{0}$ or $c_{0}$ in such a way that the behaviour of the $\log i t\left(q_{x t_{0}}\right)$ is as similar as possible to the estimations (A.1).

\section{Appendix B. Covariance function fitting}

We use the residuals (6) to estimate the empirical covariance function. The valid model fitted to the empirical one is a model proposed by Gneiting (2002), whose expression is

$C(h, u)=\frac{\sigma^{2}}{2^{\nu-1} \Gamma(\nu)\left(a|u|^{2 \alpha}+1\right)^{\delta+\beta / 2}}\left(\frac{c\|h\|}{\left(a|u|^{2 \alpha}+1\right)^{\beta / 2}}\right)^{\nu} K_{\nu}\left(\frac{c\|h\|}{\left(a|u|^{2 \alpha}+1\right)^{\beta / 2}}\right)$,

which depends on a vector of parameters $\boldsymbol{\theta}=\left(a, c, \alpha, \beta, \nu, \delta, \sigma^{2}\right)^{\prime}$, where $c>0$ and $a>0$ are the spatial and temporal parameters, respectively, $\nu>0$ is a spatial smoothing parameter, $\alpha \in] 0,1]$ is a temporal smoothing parameter, $\beta \in[0,1]$ is a parameter controlling the space-time interaction with $\beta=0$ for separable covariance and $\beta=1$ for complete interaction, $\sigma^{2}>0$ is the variance of the space-time process and $\delta \geq 0$ a shape parameter. In (B.1), 
$\mathcal{K}_{\nu}$ is the modified Bessel function of the second kind of order $\nu$ (Abramowitz and Stegun, 1965).

The choice of this covariance function model is motivated by its flexibility for adjustment. Theoretical details and properties of this class of covariance functions can be found in Martínez-Ruiz (2008).

\section{Acknowledgments}

This work was partially supported by grants from the MEyC (Ministerio de Educación y Ciencia, Spain, project MTM2007-62923 and project MTM2008-05152). The research by Ana Debón and Francisco Martínez-Ruiz has also been partially supported by a grant from the Generalitat Valenciana (grant No. GVPRE/2008/103).

\section{References}

Abramowitz, M. and Stegun, I. A. (1965). Handbook of Mathematical Functions. Dover, New York.

Benjamin, B. and Soliman, A. (1993). Mortality on the Move. Actuarial Education Service, Oxford.

Booth, H., Hyndman, R., Tickle, L., and de Jong, P. (2006). Lee-Carter mortality forecasting: a multi-country comparison of variants and extensions. Demographic Research, 15(9):289-310.

Booth, H., Maindonald, J., and Smith, L. (2002). Applying Lee-Carter under conditions of variable mortality decline. Population Studies, 56(3):325-336. 
Cressie, N. (1993). Statistics for Spatial Data, Revised Edition. John Wiley, New York.

Cressie, N. and Majure, J. (1997). Spatio-temporal statistical modelling of livestock waste in streams. Journal of Agricultural, Biological, and Environmental Statistics, 2(1):24-47.

Debón, A., Montes, F., and Puig, F. (2008). Modelling and forecasting mortality in Spain. European Journal of Operation Research, 189(3):624637.

Debón, A., Montes, F., and Sala, R. (2005). A comparison of parametric models for mortality graduation. Application to mortality data of the Valencia region (Spain). Statistics and Operations Research Transactions, 29(2):269-287.

Debón, A., Montes, F., and Sala, R. (2006a). A comparison of models for dinamical life tables. Application to mortality data of the Valencia region (Spain). Lifetime data analysis, 12(2):223-244.

Debón, A., Montes, F., and Sala, R. (2006b). A comparison of nonparametric methods in the graduation of mortality: application to data from the Valencia region (Spain). International Statistical Review, 74(2):215-233.

Brouhns, N., Denuit, M., and Keilegom, I. V. (2005). Bootstrapping Poisson log-bilinear model for mortality forecasting. Scandinavian Actuarial Journal, (3):212-224.

Efron, B. y Tibshirani, R. (1993). An introduction to the boostrap. Chapman \& Hall, New York \& London. 
Felipe, A., Guillén, M., and Pérez-Marín, A. (2002). Recent mortality trends in the Spanish population. British Actuarial Journal, 8(4):757-786.

Forfar, D., McCutcheon, J., and Wilkie, A. (1988). On graduation by mathematical formula. Journal of the Institute of Actuaries, 115 part I(459):1149.

Gavin, J., Haberman, S., and Verrall, R. (1993). Moving weighted average graduation using kernel estimation. Insurance: Mathematics $\&$ Economics, 12(2):113-126.

Gavin, J., Haberman, S., and Verrall, R. (1994). On the choice of bandwidth for kernel graduation. Journal of the Institute of Actuaries, 121:119-134.

Gavin, J., Haberman, S., and Verrall, R. (1995). Graduation by kernel and adaptive kernel methods with a boundary correction. Transactions. Society of Actuaries, XLVII:173-209.

Gneiting, T. (2002). Nonseparable, stationary covariance functions for spacetime data. Journal of the American Statistical Association, 97:590-600.

Guillen, M. and Vidiella-i-Anguera, A. (2005). Forecasting Spanish natural life expectancy. Risk Analysis, 25(5):1161-1170.

Hyndman, R. J. (2008). forecast: Forecasting functions for time series. R package version 1.11 .

Journel, A. G. and Huijbregts, C. J. (1978). Mining Geoestatistics. Academic Press, New York. 
Koissi, M., Shapiro, A., and Högnäs, G. (2006). Evaluating and extending the Lee-Carter model for mortality forecasting confidence interval. Insurance: Mathematics \&6 Economics, 38(1):1-20.

Lee, R. (2000). The Lee-Carter method for forecasting mortality, with various extensions and applications. North American Actuarial Journal, 4(1):8091.

Lee, R. and Carter, L. (1992). Modelling and forecasting U. S. mortality. Journal of the American Statistical Association, 87(419):659-671.

Li, S.-H., Hardy, M., y Tan, K. (2006). Uncertainty in mortality forecasting: an extensión to the classical Lee-Carter approach. Technical report, Waterloo University.

Martínez-Ruiz, F. (2008). Modelización de la función de covarianza en procesos espacio-temporales: análisis y aplicaciones. PhD thesis, Universitat de València, Spain.

Mateu, J., Montes, F. and Plaza, M. (2004). The 1970 US draft lottery revisited: a spatial analysis. JRSS Series C (Applied Statistics), 53(1):219229.

Matheron, G. (1975). Random Sets and Integral Geometry. Wiley, New York.

Pedroza, C. (2006). A bayesian forecasting model: predicting U.S. male mortality. Biostatistics, 7(4):530-550.

Pitacco, E. (2004). Survival models in dynamic context: a survey. Insurance: Mathematics \& Economics, 35(2):279-298. 
R Development Core Team (2005). R: A Language and Environment for Statistical Computing. R Foundation for Statistical Computing, Vienna, Austria. ISBN 3-900051-07-0.

Renshaw, A. (1991). Actuarial graduation practice and generalised linear models. Journal of the Institute of Actuaries, 118(II):295-312.

Renshaw, A. and Haberman, S. (2003). Lee-Carter mortality forecasting with age specific enhancement. Insurance: Mathematics $\&$ Economics, $33(2): 255-272$.

Renshaw, A. and Haberman, S. (2006). A cohort-based extension to the Lee-Carter model for mortality reduction factors. Insurance: Mathematics \& Economics, (3):556-570.

Renshaw, A. and Haberman, S. (2008). On simulation-based approaches to risk measurement in mortality with specific reference to poisson Lee-Carter modelling. Insurance: Mathematics \&f Economics, 42(2):797-816.

Tabeau, E., van den Berg Jeths, A. and Heathcote (Eds), C. (2001). A Review of Demographic Forecasting Models for Mortality. Forecasting in Developed Countries: From description to explanation. Kluwer Academic Publishers.

Turner, H. and Firth, D. (2006). Generalized nonlinear models in R: An overview of the gnm package. $\mathrm{R}$ package version $0.9-1$.

Wong-Fupuy, C. and Haberman, S. (2004). Projecting mortality trends: Recent developents in the United Kingdom and the United States. North American Actuarial Journal, 8(2):56-83. 